\title{
How malaria models relate temperature to malaria transmission
}

\author{
Torleif Markussen Lunde ${ }^{1,2,4^{*}}$, Mohamed Nabie Bayoh ${ }^{3}$ and Bernt Lindtjørn²
}

\begin{abstract}
Background: It is well known that temperature has a major influence on the transmission of malaria parasites to their hosts. However, mathematical models do not always agree about the way in which temperature affects malaria transmission.

Methods: In this study, we compared six temperature dependent mortality models for the malaria vector Anopheles gambiae sensu stricto. The evaluation is based on a comparison between the models, and observations from semi-field and laboratory settings.

Results: Our results show how different mortality calculations can influence the predicted dynamics of malaria transmission.

Conclusions: With global warming a reality, the projected changes in malaria transmission will depend on which mortality model is used to make such predictions.
\end{abstract}

Keywords: Anopheles gambiae sensu stricto, Climate, Temperature, Mathematical model

\section{Background}

Since the 1950s, near-surface global temperatures have increased by about $0.5-0.6^{\circ} \mathrm{C}[1]$, and it is likely that temperatures will continue to increase over the next century [2]. Model predictions, reported widely in climate policy debates, project that a warmer climate could increase malaria caused by the parasites Plasmodium falciparum and $P$. vivax in parts of Africa [3]. Malaria is transmitted by mosquitoes of the Anopheles genus, with Anopheles gambiae s.s., An. arabiensis and An. funestus being the dominant vector species in Africa [4,5].

These projections rely on knowledge about how the malaria parasite and anopheline vectors respond to changes in temperature. While a lot is known [6] about how parasite development is influenced by temperature [7], the same cannot be said for mosquitoes. In addition to temperature, humidity [8,9], breeding site formation [10], and competition between mosquitoes [11,12] are important factors controlling the number of vectors at any time.

\footnotetext{
*Correspondence: torleif.lunde@cih.uib.no

1 Bjerknes Centre for Climate Research, University of Bergen, Norway

${ }^{2}$ Centre for International Health, University of Bergen, Norway

Full list of author information is available at the end of the article
}

Climate predictions about humidity and precipitation are more uncertain than temperature projections. Therefore, it is of interest to see if a consensus exists between different malaria models about how temperature alone influences malaria transmission. In the past, studies have suggested that the optimal temperature for malaria transmission is between 30 and $33^{\circ} \mathrm{C}$ [13-15].

Here, we compare six mortality models (Martens 1, Martens 2, Bayoh-Ermert, Bayoh-Parham, BayohMordecai and Bayoh-Lunde) to reference data (control) for Anopheles gambiae s.s., and show how these models can alter the expected consequences of higher temperatures. The main purpose of the study is to show if there are any discrepancies between the models, with consequences for the ability of projecting the impact of temperature changes on malaria transmission.

We have focused on models that have been designed to be used on a whole continent scale, rather than those that focus on local malaria transmission $[10,16,17]$.

\section{Methods}

\section{Survival models}

Six different parametrization schemes have been developed to describe the mortality rates for adult An. gambiae s.s.. These schemes are important for estimating the 
temperature at which malaria transmission is most efficient. The models can also be used as tools to describe the dynamics of malaria transmission. In all of the equations presented in this paper, temperature, $T$ and $T_{\text {air }}$ are in ${ }^{\circ} C$.

\section{Martens 1}

The first model, which is called Martens scheme 1 in Ermert et al. [18], and described by Martens et al. [19-21], is derived from three points, and shows the relationship between daily survival probability $(p)$ and temperature $(T)$. This is a second order polynomial, and is, mathematically, the simplest of the models.

$$
p(T)=-0.0016 \cdot T^{2}+0.054 \cdot T+0.45
$$

\section{Martens 2}

In 1997 Martens [21] described a new temperaturedependent function of daily survival probability. This model has been used in several studies [13,14,22,23]. In the subsequent text this model is named Martens 2. Numerically, this is a more complex model than Martens 1 , and it increases the daily survival probability at higher temperatures.

$$
p(T)=e^{-\frac{1}{-4.4+1.31 \cdot T-.03 \cdot T^{2}}}
$$

\section{Bayoh-Ermert}

In 2001, Bayoh carried out an experiment where the survival of $A n$. gambiae s.s. under different temperatures (5 to 40 in $5^{\circ} \mathrm{C}$ steps) and relative humidities (RHs) (40 to 100 in $20 \%$ steps) was investigated [24]. This study formed the basis for three new parametrization schemes. In the naming of these models, we have included Bayoh, who conducted the laboratory study, followed by the author who derived the survival curves.

In 2011, Ermert et al. [18] formulated an expression for Anopheles survival probability; however, RH was not included in this model. In the text hereafter, we name this model Bayoh-Ermert. This model is a fifth order polynomial.

Overall, this model has higher survival probabilities at all of the set temperatures compared with the models created by Martens.

$$
\begin{aligned}
p(T)= & -2.123 \cdot 10^{-7} \cdot T^{5}+1.951 \cdot 10^{-5} \cdot T^{4} \\
& -6.394 \cdot 10^{-4} \cdot T^{3}+8.217 \cdot{ }^{-3} \cdot T^{2} \\
& -1.865 \cdot 10^{-2} \cdot T+7.238 \cdot 10^{-1}
\end{aligned}
$$

\section{Bayoh-Parham}

In 2012, Parham et al. [25] (designated Bayoh-Parham in subsequent text) included the effects of relative humidity and parametrized survival probability using the expression shown below. This model shares many of the same characteristics as the Bayoh-Ermert model. The mathematical formulation is similar to the Martens 2 model, but constants are replaced by three terms related to $\mathrm{RH}$ $\left(\beta_{0}, \beta_{1}, \beta_{2}\right)$.

$$
p(T, R H)=e^{-\left(T^{2} \cdot \beta_{2}+T \cdot \beta_{1}+\beta_{0}\right)^{-1}}
$$

where $\beta_{0}=0.00113 \cdot R H^{2}-0.158 \cdot R H-6.61, \beta_{1}=$ $-2.32 \cdot 10^{-4} \cdot R H^{2}+0.0515 \cdot R H+1.06$, and $\beta_{2}=4 \cdot 10^{-6}$. $R H^{2}-1.09 \cdot 10^{-3} \cdot R H-0.0255$.

For all models reporting survival probability, we can rewrite $p$ to mortality rates, $\beta$ according to:

$$
\beta=-\ln (p)
$$

\section{Bayoh-Mordecai}

Recently, Mordecai et al. [26] re-calibrated the Martens 1 model by fitting an exponential survival function to a subset of the data from Bayoh and Lindsay [24]. They used the survival data from the first day of the experiment and one day before the fraction alive was 0.01 . Six data points were used for each temperature.

$$
p(T)=-0.000828 \cdot T^{2}+0.0367 \cdot T+0.522
$$

\section{Bayoh-Lunde}

From the same data [24], Lunde et al. [27], derived an agedependent mortality model that is dependent on temperature, RH, and mosquito size. This model assumes nonexponential mortality as observed in laboratory settings [24], semi-field conditions [28], and in the field [29]. In the subsequent text we call this model Bayoh-Lunde. The four other models use the daily survival probability as the measure, and assume that the daily survival probability is independent of mosquito age. The present model calculates a survival curve $(\varpi)$ with respect to mosquito age. Like the Bayoh-Parham model, we have also varied the mosquito mortality rates according to temperature and $\mathrm{RH}$.

Because mosquito size is also known to influence mortality $[8,9,30,31]$, we applied a simple linear correction term to account for this. In this model, the effect of size is minor compared with temperature and relative humidity. The survival curve, $\varpi$, is dependent on a shape and scale parameter in a similar manner as for the probability density functions. The scale of the survival function is dependent on temperature, $\mathrm{RH}$, and mosquito size, while the scale parameter is fixed in this paper.

The mortality rate, $\beta_{n}(T, R H$, size) (equation 7$)$ is fully described in Additional file 1, with illustrations in Additional files 2, and 3 .

$$
\beta_{n}(T, R H, \text { size })=\frac{\ln \left(\frac{\varpi_{N, m_{t_{2}}}}{\bar{\varpi}_{N, m_{t_{1}}}}\right)}{\Delta t}
$$

\section{Biting rate and extrinsic incubation period}

The equations used for the biting rate, $G(T)$, and the inverse of the extrinsic incubation period (EIP, $p f$ ) are described in Lunde et al. [27]. For convenience, 
these equations and their explanations are provided in Additional file 1. The extrinsic incubation period was derived using data from MacDonald [7], while the biting rate is a mixture of the degree day model by Hoshen and Morse [32], and a model by Lunde et al. [27]. Since our main interest in this research was to examine how mosquito mortality is related to temperature in models, we used the same equation for the gonotrophic cycle for all of the mortality models. If we had used different temperature-dependent gonotrophic cycle estimates for the five models, we would not have been able to investigate the effect of the mortality curves alone.

\section{Malaria transmission}

We set up a system of ordinary differential equations (ODEs) to investigate how malaria parasites are transmitted to mosquitoes. Four of the mortality models (equations 1, 2, 3, and 4) are used in a simple compartment model that includes susceptible $(S)$, infected $(E)$ and infectious mosquitoes $(I)$ (equation 8):

$$
\begin{aligned}
& \frac{d S}{d t}=-\left(\beta+G(T) \cdot H_{i}\right) \cdot S \\
& \frac{d E}{d t}=\left(G(T) \cdot H_{i}\right) \cdot S-(\beta+p f) \cdot E \\
& \frac{d I}{d t}=p f \cdot E-\beta \cdot I
\end{aligned}
$$

where $H_{i}$ is the fraction of infectious humans, which was set to $0.01 . G(T)$ is the biting rate, and $p f$ is the rate at which sporozoites develop in the mosquitoes. The model is initialized with $S=1000, E=I=0$ and integrated for 150 days with a time step of 0.5 . As the equations show, there are no births in the population, and the fraction of infectious humans is held constant during the course of the integration. This set-up ensures that any confounding factors are minimized, and that the results can be attributed to the mortality model alone.

Because the Lunde et al. [27] (Bayoh-Lunde) mortality model also includes an age dimension, the differential equations must be written taking this into account. Note that the model also can be used in equation 8 if we allow $\beta$ to vary with time.

We separate susceptible $(S)$, infected $(E)$ and infectious $(I)$, and the subscript denotes the age group. In total there are 25 differential equations, but where the equations are similar, the subscript $n$ has been used to indicate the age group.

Formulating the equation this way means we can estimate mosquito mortality for a specific age group. We have assumed that mosquito biting behaviour is independent of mosquito age; this formulation is, therefore, comparable to the framework used for the exponential mortality models.
The number of infectious mosquitoes is the sum of $I_{n}$, where $n=2, \ldots, 9$.

$$
\begin{aligned}
\frac{d S_{1}}{d t}= & -\left(\beta_{1}+a_{1}\right) \cdot S_{1} \\
\frac{d S_{n}}{d t}= & a_{n-1} \cdot S_{1}-\left(\beta_{n}+a_{n}+G(T) \cdot H_{i}\right) \cdot S_{n} \\
& n=2,3, \ldots, 9 \\
\frac{d S_{n}}{d t}= & G(T) \cdot H_{i} \cdot S_{2}-\left(\beta_{2}+a_{2}+p f\right) \cdot E_{2} \\
\frac{d E_{n}}{d t}= & G(T) \cdot H_{i} \cdot S_{n}+a_{n-1} \cdot E_{n-1} \\
& -\left(\beta_{n}+a_{n}+p f\right) \cdot E_{n} \\
& n=3,4, \ldots, 9 \\
\frac{d I_{2}}{d t}= & p f \cdot E_{2}-\left(\beta_{2}+a_{2}\right) \cdot I_{2} \\
\frac{d I_{n}}{d t}= & p f \cdot E_{n}+a_{2} \cdot I_{n-1}-\left(\beta_{n}+a_{n}\right) \cdot I_{n} \\
& n=3,4, \ldots, 9
\end{aligned}
$$

Age groups for mosquitoes $(m)$ in this model are $m_{1}=$ $[0,1], m_{2}=(2,4], m_{3}=(5,8], m_{4}=(9,13], m_{5}=$ $(14,19], m_{6}=(20,26], m_{7}=(27,34], m_{8}=(35,43]$, $m_{9}=(44, \infty]$ days, and coefficients $a_{n}$, where $n=$ $1,2, \ldots, 9$, are $1.000,0.500,0.333,0.250,0.200,0.167$, $0.143,0.125,0.067$. The rationale behind these age groups is that as mosquitoes become older, there is a greater tendency of exponential mortality compared to younger mosquitoes.

This model has initial conditions $S_{1}=1000$, and all other 0 .

A note on the use of ODEs and rate calculations can be found in Additional file 4.

\section{Validation data}

To validate the models, we used the most extensive data set available on mosquito survival [24] under different temperatures ( 5 to 40 by $5^{\circ} \mathrm{C}$ ) and RHs ( 40 to 100 by 20\%) [24]; it is the same data that the Bayoh-Ermert, BayohParham and Bayoh-Lunde models were derived from. These data describe the fraction of live mosquitoes $\left(f_{a}\right)$ at time $t$, which allows us to validate the models over a range of temperatures. Because three of the models used the Bayoh and Lindsay data to develop the survival curves, this comparison is unrealistic for Martens models.

Hence, to account for this we have used three independent data sets to validate the fraction of infectious mosquitoes and the mosquito survival curves.

Scholte et al. (Figure two in [33]) published a similar data set, but this was based on a temperature of $27 \pm 1^{\circ} \mathrm{C}$ 
and a RH of $80 \pm 5 \%$, whereas Afrane et al. (Figure two in [28]) used mean temperatures of 21.5 to 25.0 and RHs of $40-80 \%$. Use of these data sets will allow us to complement the validation to determine if the patterns of malaria transmission are consistent with that of the control (Table 1). In addition to the data from Scholte et al. [33], we also found the following data set, which is suitable for validation of the survival curves but not the transmission process itself, because the data does not show the survival curve until all of the mosquitoes are dead [Kikankie, Master's thesis (Figures three to eight, chapter $3,25^{\circ} \mathrm{C}, 80 \% \mathrm{RH}$ ) [34]]. These results are also shown in Table 1 . The additional validation only gives information about the model quality between 21 , and $27^{\circ} \mathrm{C}$; however, it serves as an independent model evaluation to determine if the results are consistent and independent of the data set used to validate the models.

Using the data from Bayoh and Lindsay, Afrane et al. or Scholte et al. [33], we can calculate the fraction of mosquitoes that would become infectious at time $t$, using equation 8. We replace $\beta$ with the time-dependent $\beta(t)$, which is a time varying mortality rate. This approach was used for the data from [24] and [33].

$$
\beta(t)=-\ln \left(\frac{f_{a}\left(t+\frac{1}{2}\right)}{f_{a}\left(t-\frac{1}{2}\right)}\right)
$$

$\beta(t)$ is linearly interpolated at times with no data. The reference data from Bayoh and Lindsay [24] are hereafter designated as the control data in the subsequent text, whereas data from Scholte et al. [33] is called Scholte in Table 1. Table 1 also shows the skill scores of the mortality model alone (for the figures in Additional file 3).

Because some of the schemes do not include $\mathrm{RH}$, we have displayed the mean number of infectious mosquitoes, $I$, for schemes that do include it. For the validation statistics, RH has been included. However, for schemes where the RH has not been taken into account, single realization at all humidities has been employed.

\section{Validation statistics}

Skill scores $(S)$ are calculated following Taylor [35]:

$$
S_{s}=\frac{4 \cdot(1+r)^{4}}{\left(\hat{\sigma}_{f}+1 / \hat{\sigma}_{f}\right)^{2} \cdot\left(1+r_{0}\right)^{4}}
$$

where $r$ is the Pearson correlation coefficient, $r_{0}=1$ is the reference correlation coefficient, and $\hat{\sigma}_{f}$ is the variance of the control over the standard deviation of the model $\left(\sigma_{f} / \sigma_{r}\right)$. This skill score will increase as a correlation increases, as well as increasing as the variance of the model approaches the variance of the model.

The Taylor diagram used to visualize the skill score takes into account the correlation (curved axis), ability to represent the variance ( $\mathrm{x}$ and $\mathrm{y}$ axis), and the root mean square.

Another important aspect is determining at which temperatures transmission is most efficient. If mosquitoes have a peak of infectiousness at, for example, $20^{\circ} \mathrm{C}$ in one model, temperatures above this will lead to a smaller fraction of mosquitoes becoming infectious. A different model might set this peak at $27^{\circ} \mathrm{C}$, so that at temperatures from $20-27^{\circ} \mathrm{C}$, the fraction of infectious mosquitoes will increase, followed by a decrease at higher temperatures. Isolating the point at which the mosquitoes are the most efficient vectors for malaria parasites is important for assessing the potential impacts of climate change. To show the differences between the models, we report the temperature where the maximum efficiency for producing infectious mosquitoes was observed. This can be done by maximizing equation 12 .

$$
\arg \max _{T \in[10,40]} \int_{t=0}^{\infty} I d t
$$

For the transmission process we also report Akaike information criterion (AIC) [36] from a generalized linear model with normal distribution. Since the observations are not independent, and residuals do not follow a normal distribution, we sample 100 values from the simulations 1000 times. We set the probability of sampling $y_{i, j}$ equal

Table 1 Skill scores

\begin{tabular}{lcrcccc}
\hline & Control & AIC Control & Scholte & AF & BL mortality model & SK mortality model \\
\hline Martens 1 & 0.01 & $76(56,96)$ & 0.00 & 0.03 & 0.36 & 0.25 \\
Martens 2 & 0.38 & $9(-14,30)$ & 0.55 & 0.37 & 0.54 & 0.45 \\
Martens 3 & 0.65 & $-38(-75,-9)$ & 0.53 & 0.77 & 0.65 & 0.52 \\
Bayoh-Ermert & 0.27 & $30(1,58)$ & 0.16 & 0.43 & 0.79 & 0.56 \\
Bayoh-Parham & 0.16 & $26(-11,55)$ & 0.05 & 0.31 & 0.79 & 0.59 \\
Bayoh-Lunde & 0.90 & $-111(-148,-81)$ & 0.83 & 0.94 & 0.90 & 0.81 \\
Bayoh-Mordecai & 0.62 & $-53(-82,-29)$ & 0.58 & 0.70 & 0.57 & 0.49 \\
\hline
\end{tabular}

Skill scores as defined in equation 11. "Control" represents the validation of infectious mosquitoes using the data from Bayoh and Lindsay [24], "Scholte" [33] represents the validation of infectious mosquitoes using the data from Scholte, "AF" represents the validation of infectious mosquitoes using the data from Afrane, "BL mortality model" represents the validation of the mortality model using the data from Bayoh and Lindsay [24], and "SK mortality model" represents the validation of the mortality model using data from Scholte [33] and Kikankie [34]. "AIC control" is Akaike information Criterion for "Control" (95\% confidence interval). 
to normalized (sum $=1$ ) fraction of infected mosquitoes of the control. This method allow us to generate a model with normally distributed, non-correlated errors. Median AIC, with 95\% confidence intervals are reported in Table 1.

\section{Results}

Figure 1 shows the percentage of infectious mosquitoes plotted against time (days) $(\mathrm{x})$ and temperature (y). The control shows that the most efficient transmission occurs at $25^{\circ} \mathrm{C}$, while the maximum percentage of infectious mosquitoes at any time is 1.1. We found that the Martens 1 and 2 models both underestimate the fraction of infectious mosquitoes, while the Bayoh-Ermert and Bayoh-Lunde models had comparable values. While the Bayoh-Parham model affords similar values at $40 \% \mathrm{RH}$, it overestimates the fraction of infectious mosquitoes at higher RHs (Additional file 3). There are also substantial differences at which the temperatures for transmission are most efficient.

While Martens 1 has the most efficient transmission at $20.4^{\circ} \mathrm{C}$, Martens 2 and Bayoh-Ermert show the transmission efficiency peaking at 26.8 and $27.5^{\circ} \mathrm{C}$. Both the control and Bayoh-Lunde models peak at $25^{\circ} \mathrm{C}$, as measured according to equation 12, Bayoh-Parham peaks at $26.3^{\circ} \mathrm{C}$, and Bayoh-Mordecai peaks at $24.4^{\circ} \mathrm{C}$ (Figure 2).

The numerical solution of the Bayoh-Ermert mortality model also reveals that it has problems related to enhanced mosquito longevity at all of the selected temperatures; this effect was especially pronounced around $20^{\circ} \mathrm{C}$. We also found that the Bayoh-Parham model has issues with prolonged mosquito survival.

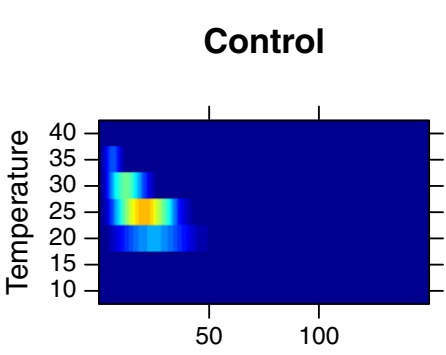

Martens 2

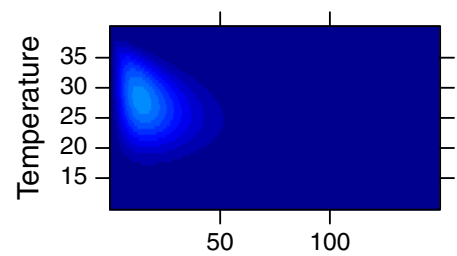

Bayoh-Parham

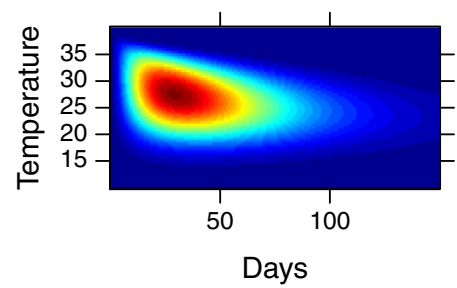

Martens recalibrated

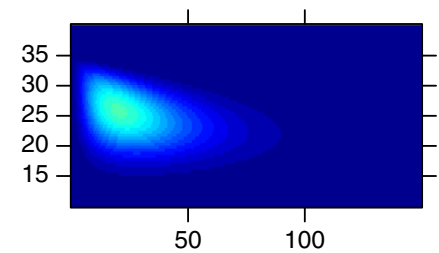

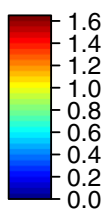
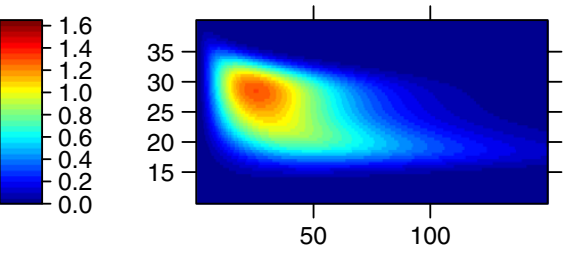

Bayoh-Lunde
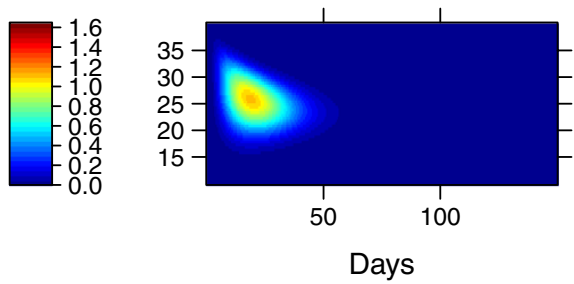

Bayoh-Mordecai

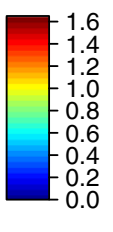

Bayoh-Ermert

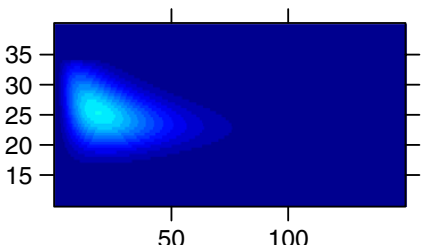

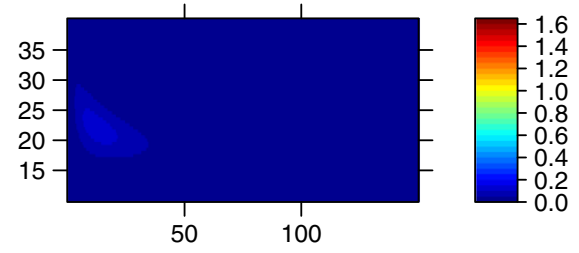
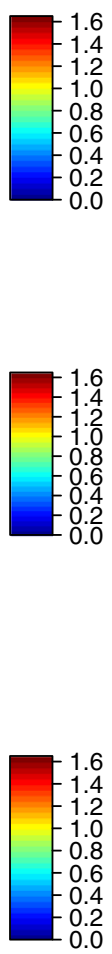

Figure 1 The percentage of infectious mosquitoes over time and temperature. 


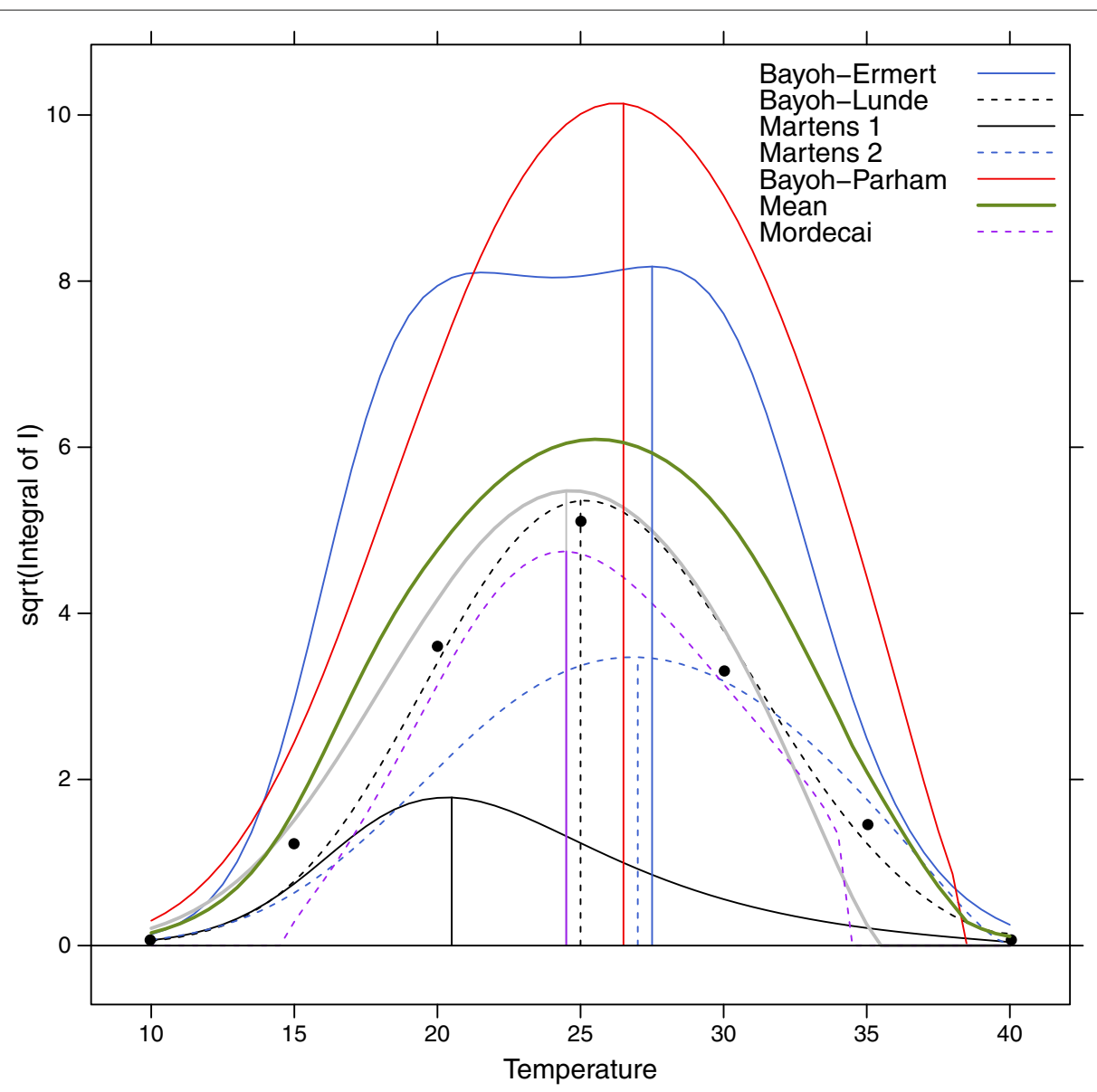

Figure 2 Integral of infectious mosquitoes over temperature. Models: Bayoh-Ermert (blue solid line), Martens 1 (black solid line), Martens 2 (blue dashed line), Martens 3 (grey solid line), Bayoh-Parham (red solid line), Lunde (black dashed line), and the mean value of the five models (green thick solid line). Black dots indicate the results for the control, and vertical lines show the temperature at which the maximum can be found (equation 12).

To evaluate the skill of the models, with emphasis on spatial patterns and variance, we investigated the skill score that was defined in equation 11 . The standard deviation, root mean square and correlation coefficient are summarized in a Taylor diagram (Figure 3). Skill scores closer to 1 are a sign of better performance from a model (Table 1).

When validating the transmission process using the data from Bayoh and Lindsay (Table 1, column 1), the majority of the penalty for the Martens 1 and 2 models was due to the low variance, indicating that the mortality is set too high compared with the reference. Further analysis found that the Bayoh-Ermert model correlated poorly with the reference, and the variance, $\hat{\sigma}_{f}$, was too high. The BayohParham model also suffered from low correlation, as well as too high variance. Overall, the Bayoh-Lunde model has the highest skill score, followed by the Bayoh-Mordecai model. The patterns are consistently independent of the data used to validate the models with respect to the malaria transmission process. Validation of the survival curves alone, and their relationship with the transmission process, is discussed in the next section.

The relatively simple Martens 2 model ranked third among the models. We re-calibrated [37,38] the model using the data from Bayoh and Lindsay. The re-calibrated model (equation 13) generated a skill score of 0.65 (for the transmission process). In addition, Martens 2 was most efficient at $24.5^{\circ} \mathrm{C}$. The Martens 3 model can be used for temperatures between 5 and $35^{\circ} \mathrm{C}$.

$$
p(T)=e^{-\frac{1}{-4.31564+2.19646 \cdot T-0.058276 \cdot T^{2}}}
$$

The newly calibrated Martens 2 model (hereafter called Martens 3), can be seen in Figure 2; the skill scores are reported in Table 1.

To investigate how sensitive the results of the Mordecai et al. [26] analysis are to the choice of mortality model, we calculated the optimal temperature for malaria transmission using their full temperature-sensitive malaria $R_{0}$ model (equation 2 in [26]). The mortality rate, $\mu(T)$, was 


\section{Taylor Diagram}

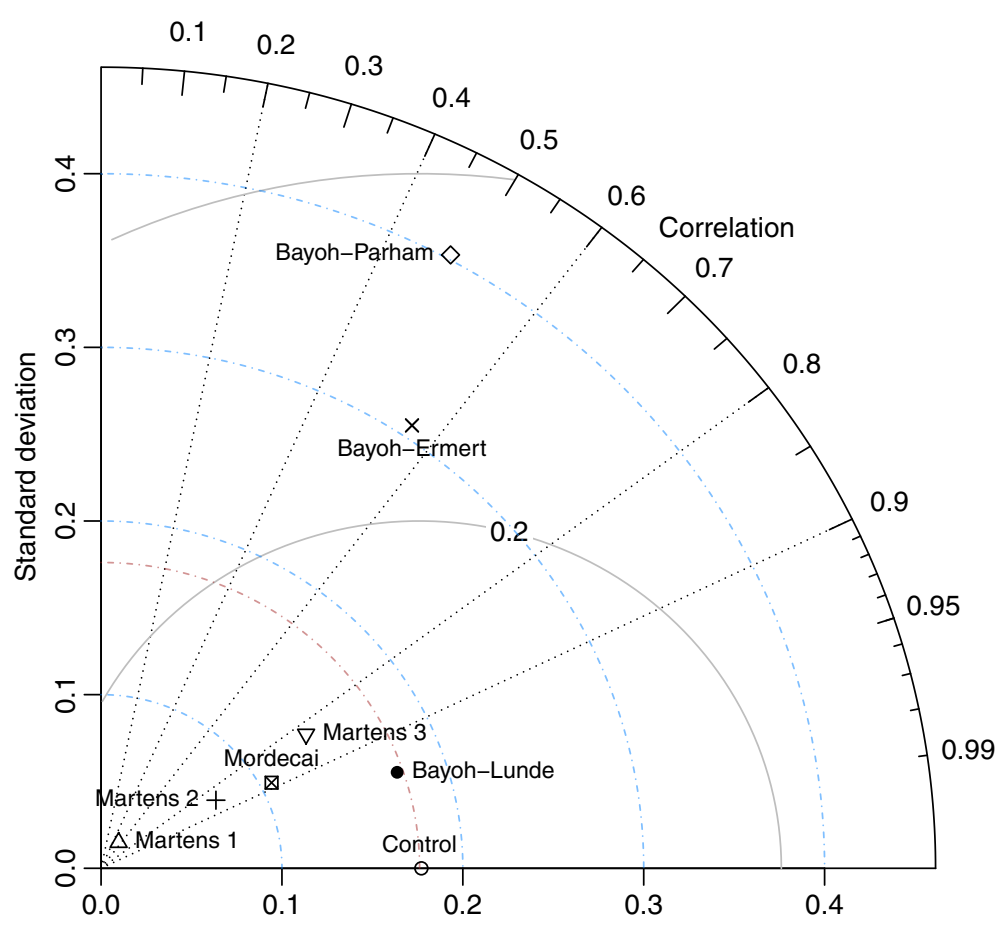

Figure 3 Taylor diagram. The model names are indicated next to the symbols. The $x$ and $y$ axes represent the standard deviations, the curved grey lines are the root mean square, while the dashed lines represent the Pearson correlation coefficient.

replaced with $-\ln (p(T))$ from the exponential models. Population density $(N)$, and recovery rate, $r$, were set to 1 , since these do not influence the optimal temperature for malaria transmission. The results can be seen in Table 2 . Relative differences between the two methods is in the range from $1-11 \%$ (Table 2). Figure 4 shows $R_{0}$ according to temperature (with $N=1, r=1$ ) for the exponential models. The maximum $R_{0}$ ranges from 10 (Martens 1 ) to 206 (Bayoh-Parham).

Table 2 Optimal temperature for malaria transmission

\begin{tabular}{lccc}
\hline & This paper & $\begin{array}{c}\boldsymbol{R}_{\mathbf{0}} \text { from Mordecai } \\
\text { et al. }\end{array}$ & $\begin{array}{c}\text { Relative } \\
\text { difference \% }\end{array}$ \\
\hline Martens 1 & 20.4 & 23.0 & 11.98 \\
Martens 2 & 26.8 & 27.0 & 0.74 \\
Martens 3 & 24.7 & 26.0 & 5.13 \\
Bayoh-Ermert & 27.5 & 27.2 & 1.10 \\
Bayoh-Parham & 26.3 & 26.9 & 2.26 \\
Bayoh-Lunde & 25.2 & & \\
Bayoh-Mordecai & 24.4 & 25.6 & 4.80 \\
\hline
\end{tabular}

Optimal temperature calculated using the methods in this paper, and by using methodology in Mordecai et al. [26].

\section{Discussion and conclusions}

The relationship between sporozoite development and the survival of infectious mosquitoes at different temperatures is poorly understood; therefore, any model projections relating the two should be interpreted with care. The Martens 2 and Bayoh-Ermert models suggest that areas of the world where temperatures approach $27^{\circ} \mathrm{C}$ could experience more malaria. Martens 3, Bayoh-Mordecai, and our model (Bayoh-Lunde) suggests that transmission is most efficient at around $25^{\circ} \mathrm{C}$. The Martens 1 model peaks at $20.4^{\circ} \mathrm{C}$, and Bayoh-Parham at $26.3^{\circ} \mathrm{C}$ (Figure 1). None of the models, except Bayoh-Lunde, capture all of the characteristics of the reference data, however.

Table 1 also shows the skill score for the mortality model alone. Both the Bayoh-Parham and the BayohErmert models have good representations of the survival curves. However, the nature of the exponential mortality curves gives them the choice of rapid mortality giving a reasonable, but underestimated, transmission process (Martens 2), or a good fit to the survival curves, which in turn makes the mosquitoes live too long, resulting in a poor transmission process (Bayoh-Parham and Bayoh-Ermert). Because the Bayoh-Lunde model offers a fair description of the survival curves as well as an age structure in the differential equations, we consider that the transmission process is well described. The Martens 


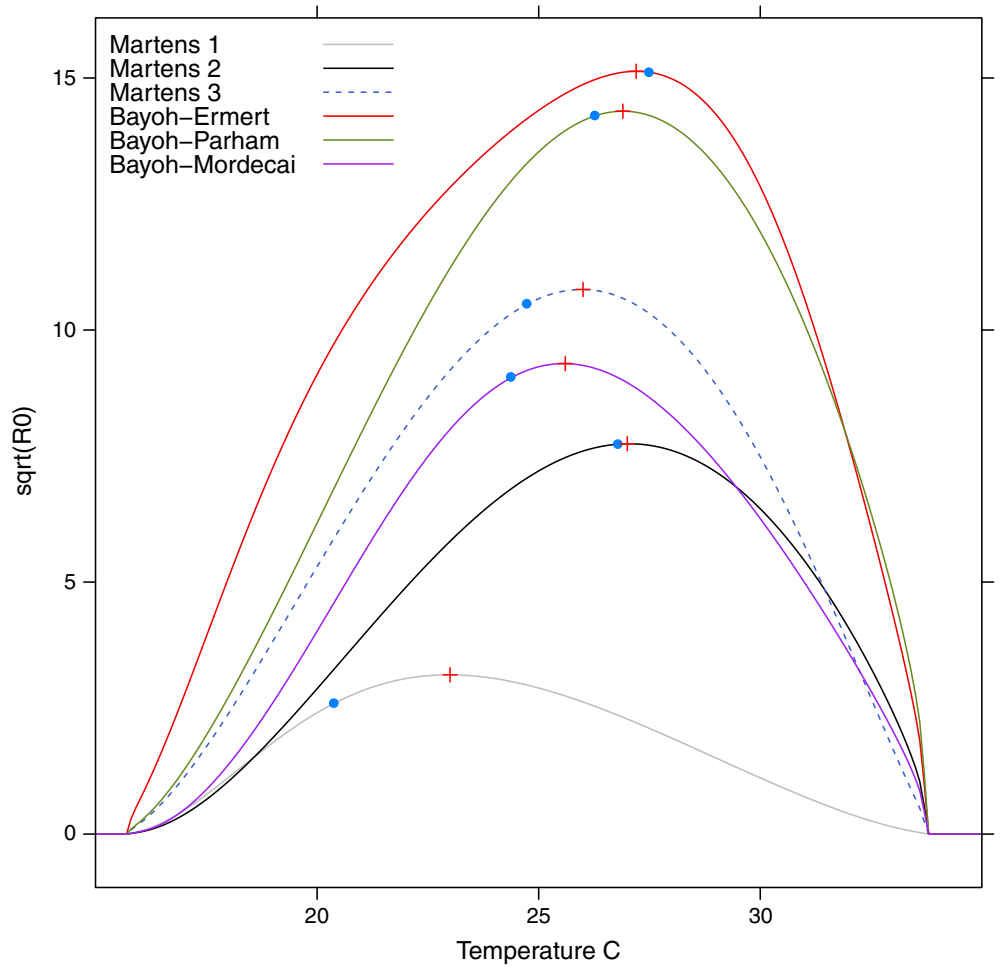

Figure $4 R_{0}$ as a function of temperature calculated using equation 2 in Mordecai et al. [26], but with different mortality models. Blue dots represent optimal temperatures using the methods in this paper, and red crosses is the optimal temperature using the methods from Mordecai et al. [26].

1 and 2, Bayoh-Ermert, Bayoh-Mordecai and BayohParham models all assume constant mortality rates with age, and would, therefore, not benefit from being solved in an age-structured framework.

The Martens 1 model has been used in several studies [19-21], with the latest appearance by Gething et al. in this journal [39]. Considering the poor skill of the Martens 1 model, the validity, or etiology, of results presented in these papers should be carefully considered.

It is likely that regions with temperatures below $18^{\circ} \mathrm{C}$, as is typical for the highland areas of East and Southern Africa, which are too cold for malaria transmission, might experience more malaria if their temperatures increase. However, malaria transmission in the future will be dependent on many other factors such as poverty, housing, access to medical care, host immunity and malaria control measures.

Most countries in Sub-Saharan Africa have annual mean temperatures between 20 and $28^{\circ} \mathrm{C}$. In these areas, linking past and future temperature fluctuations to changes in malaria transmission is challenging. Our data suggest that one way to reduce this uncertainty is to use age-structured mosquito models. These models produce results that agree with the observed data, and nonexponential mosquito mortality has been demonstrated in several studies [33,40-42], although the true nature of mosquito survival in the field is not fully elucidated. The newly calibrated Martens 2 model described here also produces acceptable results. If simplicity is a goal in itself [43], models that assume exponential mortality will still have utility. To believe in projections of the potential impact of long-term, large-scale climate changes, it is crucial that models have an accurate representation of malaria transmission, even at the cost of complexity. For studies of malaria transmission at village level, other approaches might be more suitable $[10,16,44,45]$.

\section{Additional files}

Additional file 1: Details of the Bayoh-Lunde model, mosquito biting rate, and parasite extrinsic incubation period.

Additional file 2: This file shows how $\zeta$ can be used to change the shape of the Bayoh-Lunde survival curve. The black line is the reference data, while the red line represents the Bayoh-Lunde survival curve.

Temperature, relative humidity (as a fraction from 0 to 1 ), and $\zeta$ are given in the panel strips.

Additional file 3: Survival curves for all of the models investigated in this study plotted at different temperatures and relative humidities.

The figure on page two shows the legend as well as an example of non-exponential mortality.

Additional file 4: A note on the use of ordinary differential equations, age structure (with an example), and rate calculations. 
Abbreviations

BL: Bayoh and Lindsay; EIP: Extrinsic incubation period; ODEs: Ordinary differential equations; SK: Scholte and Kikankie.

\section{Competing interests}

The authors declare that they have no competing interests.

\section{Authors' contributions}

The work presented here was carried out in collaboration between all of the authors. BL, MNB and TML defined the research theme. MNB provided the data for the control. TML designed the methods and experiments, did the model runs, analysed the data, interpreted the results, and wrote the paper. All authors read and approved the final version of the manuscript.

\section{Acknowledgements}

This work was made possible by grants from The Norwegian Programme for Development, Research and Education (NUFU) and the University of Bergen. Our thanks go to Asgeir Sorteberg for commenting on the manuscript, and three anonymous reviewers for their constructive comments, which helped us to improve the manuscript

\section{Author details}

${ }^{1}$ Bjerknes Centre for Climate Research, University of Bergen, Norway. ${ }^{2}$ Centre for International Health, University of Bergen, Norway. ${ }^{3} \mathrm{KEMRI} / \mathrm{CDC}$ Research and Public Health Collaboration, Kisumu, Kenya. ${ }^{4}$ Bjerknes Centre for Climate Research, Uni Research, Norway.

Received: 5 October 2012 Accepted: 15 January 2013

Published: 18 January 2013

\section{References}

1. Hansen J, Sato M, Ruedy R: Perception of climate change. Proc Natl Acad Sci USA 2012, 109:E2415-2423.

2. Intergovernmental Panel on Climate Change: Fourth Assessment Report: Climate Change 2007: Working Group I Report: The Physical Science Basis. Geneva: IPCC; 2007

3. Gething PW, Smith DL, Patil AP, Tatem AJ, Snow RW, Hay SI: Climate change and the global malaria recession. Nature 2010, 465:342-345.

4. Sinka ME, Bangs MJ, Manguin S, Rubio-Palis Y, Chareonviriyaphap T, Coetzee M, Mbogo CM, Hemingway J, Patil AP, Temperley WH, Gething PW, Kabaria CW, Burkot TR, Harbach RE, Hay SI: A global map of dominant malaria vectors. Parasit Vectors 2012, 5:69.

5. Sinka ME, Bangs MJ, Manguin S, Coetzee M, Mbogo CM, Hemingway J, Patil AP, Temperley WH, Gething PW, Kabaria CW, Okara RM, Van Boeckel T, Godfray HCJ, Harbach RE, Hay SI: The dominant Anopheles vectors of human malaria in Africa, Europe and the Middle East: occurrence data, distribution maps and bionomic precis. Parasit Vectors 2010, 3:117.

6. Paaijmans KP, Blanford S, Chan BHK, Thomas MB: Warmer temperatures reduce the vectorial capacity of malaria mosquitoes. Biol Lett 2012 , 8:465-468.

7. MacDonald G: Dynamics of Tropical Disease. London: Oxford University Press; 1973

8. Gray EM, Bradley TJ: Physiology of desiccation resistance in Anopheles gambiae and Anopheles arabiensis. Am J Trop Med Hyg 2005, 73:553-559.

9. Fouet C, Gray E, Besansky NJ, Costantini C: Adaptation to aridity in the malaria mosquito Anopheles gambiae: chromosomal inversion polymorphism and body size influence resistance to desiccation. PLoS One 2012, 7:e34841.

10. Bomblies A, Duchemin JB, Eltahir EAB: Hydrology of malaria: Model development and application to a Sahelian village. Water Resour Res 2008, 44:W12445.

11. Paaijmans KP, Huijben S, Githeko AK, Takken W: Competitive interactions between larvae of the malaria mosquitoes Anopheles arabiensis and Anopheles gambiae under semi-field conditions in western Kenya. Acta Trop 2009, 109:124-130.

12. Kweka EJ, Zhou G, Beilhe LB, Dixit A, Afrane Y, Gilbreath TM, Munga S, Nyindo M, Githeko AK, Yan G: Effects of co-habitation between Anopheles gambiae s.s. and Culex quinquefasciatus aquatic stages on life history traits. Parasit Vectors 2012, 5:33.
13. Craig MH, Snow RW, le Sueur D: A climate-based distribution model of malaria transmission in Sub-Saharan Africa. Parasitol Today 1999, 15:105-111.

14. Parham PE, Michael E: Modeling the effects of weather and climate change on malaria transmission. Environ Health Perspect 2010, 118:620-626

15. Martens W: Health impacts of climate change and ozone depletion. An eco-epidemiological modelling approach. The Netherlands: Maastricht University Press; 1997.

16. Depinay JMO, Mbogo CM, Killeen G, Knols B, Beier J, Carlson J, Dushoff J, Billingsley P, Mwambi H, Githure J, Toure AM, McKenzie FE: A simulation model of African Anopheles ecology and population dynamics for the analysis of malaria transmission. Malar J 2004, 3:29.

17. White MT, Griffin JT, Churcher TS, Ferguson NM, Basanez MG, Ghani AC: Modelling the impact of vector control interventions on Anopheles gambiae population dynamics. Parasit Vectors 2011, 4:153.

18. Ermert $\mathrm{V}$, Fink AH, Jones AE, Morse AP: Development of a new version of the Liverpool Malaria Model. I. Refining the parameter settings and mathematical formulation of basic processes based on a literature review. Malar $J$ 2011, 10:35.

19. Martens W, Jetten T, Rotmans J, Niessen L: Climate change and vector-borne diseases: A global modelling perspective. Global Environmental Change 1995, 5:195-209.

20. Martens WJ, Niessen LW, Rotmans J, Jetten TH, McMichael AJ: Potential impact of global climate change on malaria risk. Environ Health Perspect 1995, 103:458-464.

21. Martens W: Health impacts of climate change and ozone depletion: an eco-epidemiological modelling approach. PhD thesis. Maastricht, Netherlands: Maastricht University; 1997.

22. Parham PE, Michael $\mathrm{E}$ : Modeling the effects of weather and climate change on malaria transmission. Environ Health Perspect 2010 118:620-626.

23. Ruiz D, Poveda G, Velez ID, Quinones ML, Rua GL, Velasquez LE, Zuluaga JS: Modelling entomological-climatic interactions of Plasmodium falciparum malaria transmission in two Colombian endemic-regions: contributions to a National Malaria Early Warning System. Malar J 2006, 5:66.

24. Bayoh N: Studies on the development and survival of Anopheles gambiae sensu stricto at various temperatures and relative humidities. PhD thesis. University of Durham; 2001.

25. Parham PE, Pople D, Christiansen-Jucht C, Lindsay S, Hinsley W, Michael E: Modeling the role of environmental variables on the population dynamics of the malaria vector Anopheles gambiae sensu stricto. Malar J 2012, 11:271.

26. Mordecai EA, Paaijmans KP, Johnson LR, Balzer C, Ben-Horin T, de Moor E, McNally A, Pawar S, Ryan SJ, Smith TC, Lafferty KD, Thrall P: Optimal temperature for malaria transmission is dramatically lower than previously predicted. Ecol Lett 2013, 16:22-30.

27. Lunde TM, Korecha D, Loha E, Sorteberg A, Lindtjørn B: A dynamic model of some malaria-transmitting anopheline mosquitoes of the Afrotropical region. I. Model description and sensitivity analysis. Malaria J 2013. in press.

28. Afrane YA, Zhou G, Lawson BW, Githeko AK, Yan G: Effects of microclimatic changes caused by deforestation on the survivorship and reproductive fitness of Anopheles gambiae in western Kenya highlands. Am J Trop Med Hyg 2006, 74:772-778

29. Harrington LC, Vermeylen F, Jones JJ, Kitthawee S, Sithiprasasna R, Edman JD, Scott TW: Age-dependent survival of the dengue vector Aedes aegypti (Diptera: Culicidae) demonstrated by simultaneous release-recapture of different age cohorts. J Med Entomol 2008 45:307-413.

30. Lyimo EO, Koella JC: Relationship between body size of adult Anopheles gambiae s.l. and infection with the malaria parasite Plasmodium falciparum. Parasitology 1992, 104:233-237.

31. Ameneshewa B, Service MW: The relationship between female body size and survival rate of the malaria vector Anopheles arabiensis in Ethiopia. Med Vet Entomol 1996, 10:170-172.

32. Hoshen M, Morse A: A model structure for estimating malaria risk. In Environmental Change and Malaria Risk: Global and Local Implications, Volume 9. Edited by Takken W, Martens P, Bogers RJ. Dordrecht, The Netherlands: Springer; 2005:10. 
33. Scholte EJ, Knols BGJ, Takken W: Infection of the malaria mosquito Anopheles gambiae with the entomopathogenic fungus Metarhizium anisopliae reduces blood feeding and fecundity. $J$ Invertebr Pathol 2006, 91:43-49.

34. Kikankie C: Susceptibility of laboratory colonies of members of the Anopheles gambiae complex to entomopathogenic fungi Beauveria bassiana. Master's thesis. Johannesburg: University of the Witwatersrand; 2009.

35. Taylor KE: Summarizing multiple aspects of model performance in a single diagram. J Geophys Res 2001, 106:7183-7192.

36. Akaike $\mathrm{H}$ : A new look at the statistical model identification. IEEE Trans Autom Control 1974, 19:716-723.

37. Byrd RH, Lu-Chen PH, Nocedal J, Zhu CY: A limited memory algorithm for bound constrained optimization. Siam J Scientific Comput 1995, 16:1190-1208.

38. R Development Core Team: R: A Language and Environment for Statistical Computing. Vienna, Austria: R Foundation for Statistical Computing; 2011

39. Gething PW, Van Boeckel TP, Smith DL, Guerra CA, Patil AP, Snow RW, Hay SI: Modelling the global constraints of temperature on transmission of Plasmodium falciparum and P. vivax. Parasit Vectors 2011, 4:92.

40. Dong Y, Morton JC, Ramirez JL, Souza-Neto JA, Dimopoulos G: The entomopathogenic fungus Beauveria bassiana activate toll and JAK-STAT pathway-controlled effector genes and anti-dengue activity in Aedes aegypti. Insect Biochem Mol Biol 2012, 42:126-132.

41. Hardstone MC, Huang X, Harrington LC, Scott JG: Differences in development, glycogen, and lipid content associated with cytochrome $\mathrm{P} 450$-mediated permethrin resistance in Culex pipiens quinquefasciatus (Diptera: Culicidae). J Med Entomol 2010, 47:188-198.

42. Glunt KD, Thomas MB, Read AF: The effects of age, exposure history and malaria infection on the susceptibility of Anopheles mosquitoes to low concentrations of pyrethroid. PLoS One 2011, 6:e24968.

43. White LJ, Maude RJ, Pongtavornpinyo W, Saralamba S, Aguas R, Van Effelterre T, Day NPJ, White NJ: The role of simple mathematical models in malaria elimination strategy design. Malar J 2009, 8:212.

44. Killeen GF, Smith TA: Exploring the contributions of bed nets, cattle, insecticides and excitorepellency to malaria control: a deterministic model of mosquito host-seeking behaviour and mortality. Trans $R$ Soc Trop Med Hyg 2007, 101:867-880.

45. Saul AJ, Graves PM, Kay BH: A cyclical feeding model for Pathogen transmission and its application to determine vectorial capacity from vector infection rates. J App/ Ecol 1990, 27:123-133.

\section{Submit your next manuscript to BioMed Central} and take full advantage of:

- Convenient online submission

- Thorough peer review

- No space constraints or color figure charges

- Immediate publication on acceptance

- Inclusion in PubMed, CAS, Scopus and Google Scholar

- Research which is freely available for redistribution

Submit your manuscript at www.biomedcentral.com/submit
C Biomed Central 